\title{
Sitzung vom 10. Februar 1913.
}

Vorsitzender: Hr. W' Will, Präsident.

Nachdem das Protokoll der letzten Sitzung genebmigt ist, teilt der Vorsitzende Folgendes mit:

Geb. Reg.-Rat Dr.-Ing.

\section{GustaV v. Brüning,}

der Generaldirektor der Farbwerke vorm. Meister, Lucius \& Brïning, ist am 8. Februar in St. Moritz am Herzschlage verschieden.

Er war der Sohn des Mitbegründers des gewaltigen Höchster Werkes und bat sich um die Leitung dieses Unternebmens während seiner erstaunlicben Futwicklung im letzten Jahrzehnt wesentliche Verdienste erworben. Für unsere Gesellschait war der Entschlafene in Jahre 1904-1905 als Ausschußmitglied tätig. Es ist ihm ein kurzer Lebenslauf beschieden gewesen; er hat ein Alter von nur 47 Jahren erreicht.

Wir wollen den ron uns gegangenen Kollegen in unserer Weise ehren.

Die Versammelten erbeben sich von ibren Sitzen.

Als ordentliche Mitglieder treten der Gesellscbaft wieder bei die HHrn.:

Vesely, Dr. Viktor, Kopernikusgasse 91, Kgl. Weinberge, Prag; Ratb, Dr. Julius, Kaiserstr. 115, Offenbacb a. M.

Als außerordentliche Mitglieder sind aufgenommen:

Hr. Blümich, E., München;

»Brettauer, H., ";

"Fuckelmann, L., " ;

"Grützuer, E., " ;

"Walther, A., $\quad$;

"Weltzien, W., ";

"Krafft, G., " ;

"Frick, Reiuh., ";
Hr. Schultze, Erast, München;

"Fraukfurter, Fr., " ;

"Welscb, Dr. A., 》 ;

"Steube, Georg, $\quad$;

"KIoß, Dr. Rud., Berlin;

»Resau, Carl, Freiburg i. B.;

»Wolff, Paul, Rostock i. M.;

»Berg, Richard, " ; 
Hr. Dy ckerboff, J., Rustock i. M.;

"Hammerschmidt, M., Rostock i. M.;

"A nuenk off, Alex., Moskau;

"Freimann, Jacob, ";

"Kulikow, Joh.,

"Simon, Alfred, Kiel;

》Beißwenger, W., 》;

》Müller, Jeau, 》;

"Anaratone, Dr. D., Settimo Torinese (Ital.);

"Balke, Dr. C. W., Urbana, Hll;

"Bister, Dr. jur., Berlin;

"Denglin, Dr. L., Moskau;

"Dunlap, Dr.F.L., ChicagoIll.;

"Ellmer, Dr. A., Berlin;

"Feulgen, Dr. med.R., BerlinCbarlotten burg;

» Kling, Dr. K., Lemberg (Galiz.);

Kock, Paul, St. Petershurg;

"Kölliker, Alfred, Zürich;

Durua y Valerdi, Prof. Dr. A., Barcelona;

"Noetzli, Dir. Dr., Aussig;

"Pfaehler, Erust, BerlinCbarlottenburg;

Hr. Tbiele Ernst, Göttingen;

"Zelluer, Dr, H., Berlin;

"Kobner, K. von, Berlin;

"Buttlar, Dr. R. T. v., Berlin;

"Karplus, Dr. H., Berlin;

- Schöffel,Dr.K.,Freiburgi.B.;

"Thole, Ferd. B., London;

* Goes, Christian, Erlangen;

"Biehler, Ferd., " ;

„Seufert, Rud., $\quad$;

"Lotz, Hans, ";

"Brunel, Dr. R. F., Brya Mawr, Pa.;

"Homann, Dr. W., Jena;

"Schö o, Ing.-Chem. L., Karlsruhe i. B.;

\Zahn, Dr.J.G., Karlsrubei. B.;

¿Sehmatloch, Dr. W., Karlsrube i. B.;

\Sonnenfeld, Dipl.-Chem.,E., Berlin-Dahlem;

"Witzel, Dr. G., Cöln;

"Karoly, Dipl.-Ing. Alex., Berlin-Charlottenburg;

》Wolf, Dr.C.G.L., Cambridge;

"Baerwindt, Dipl.-Ing., Berlin-Charlottenburg;

"Plange, Dr. Rud., Düssel- Frau Bodländer, E., Berlin. dorf;

Als außerordentlicbe Mitglieder werden vorgeschlagen die HHrn.:

Bader, Marcel, Chemiker, Heidenbein a. d. Brenz, Württbg. (durch O. N. Witt und E. Noelting);

Bilfinger, Otto, Neckarhalde 17, Tübingen (durch A. Kliegl und R. Weinland);

Goldbaum, Dr. J. S., Universität, Philadelphia, Pa. (durch H. F. Keller und E. F.Smith);

Grauer, Dr. Fr., Rechte-Bahngasse 14, Wien III (durch M. Kohn und A. Ostersetzer);

Janney, Dr. med. et chem. N. W., 154 E. $91^{\text {st Street, }}$ New York City (durch H. D. Dakin und H. W. Dudley); Maschmann, Ernst, Zimmerweg 10, Bern (durch V. Kohlschütter und F. Ephraim); 
Müble, Georg, T'echn. Hochschule, Berlin-Charlottenburg (durch C. Liebermann und H. Liebermann);

Weisbut, Fritz, Gymnasiumstr. 77, Wien XIX (durch li.

Zeruer und E. Pbilippi);

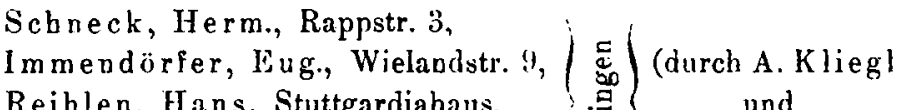

Reiblen, Hans, Stuttgardiahaus,

Pfaunenstiel, Hugo, Bursagasse 18,

Neff, Herm., Keplerstr. 191,

Konarsky, Artur, Ziegelstr. 18/19, ) (durch

Baltrusch, Walter, Königstr. 23, (Königs-)H.Klinger

Goldhorn, Hans, Assist. Unterlaak 42, (bergi.Pr.) und

Ma ecklenburg, W., Assist. Rosenstr.7, (A. Sonn);

Sandelin, Mag. phil. A. W., Hangö (Finland), (durch $O$.

Zrist, Ing.-Chem. W., Ghem. Lab. d. A scbau u.

Klingstedt, Mag.phil. F.W., Univ.Helsingfors J.Palmén);

Lemmermann, Prof. Dr., Hăndelstr. 20, Berlin NW. 23

(durch M. Delbrück und B. Lepsius);

A ufs cbläger, Geveraldirektor Dr. G., Dyaamit-A.-G. vorm.

A. Nobel \& Co., Hamburg (durch W. Will und B. Lepsius);

Oehme, Herbert, Knesebeckstr. 46/47, Berlin W. 15 (durch

C. Liebermann und H. Simonis);

Moles, Doz. Dr. E., Farmacia 11, Madrid (durch .1. Cilsares und A. Madinaveitia);

Doermer, Dr. L., Moorweg 44, Hamburg-Gr. Borstel (durcb

A. Naumano und H. Jost);

Homén, Mag. phil. Arne, Hangö (Finland) (durch O. Aschan und J. Palmén);

Strunk, Oberstabsapotbeker Dr. H., Bochumerstr. 4, Berlin

NW. 21 (durch W. Traube und R. Pschorr).

Fïr die Bibliothek sind als Geschenke eingegangen:

2161. Skita, A, Úber katalytische Reduktionen organischer Verbindungen. Chemie in Einzeldarstellungen. Bd. II. Stuttgart 1912.

2162. Ahrens, F. B. und Hinrichsen, F. W., Einführung in die praktische Chemie. Stuttgart 1912.

2163. Hinrichsen, F. W., Das Materialprüfungswesen. Stuttgart 1912.

2164. Feitler, S., Die Zuckerfabrikation. Kurzgefaßtes Iehrbuch für Studierende, Beamle und Praktiker. Wien und Leipzig 1913.

2165. Tschugaeff, L. A., Das periodische System der chemischen Elemente. St. Petersburg 1913. (Russisch.) 
2166. Dakin, H. D., Oxidations and Reductions in the Animal Body. London 1912.

2167. Söderbaum, H. G., Jac. Berzelius Lettres II. Correspondance entre Berzelius et Sir Humphry Davy (1808-1825). Upsala 1912.

2168. Rinn e, F., Elementare Anleitung zu krystallographisch-optischen Untersuchungen. 2. Aufl. Jeipzig 1912.

2169. Giolitti, F, La Cementazione dell' Acciaio. Turin 1912.

2170. Kondakow, I. L., Der synthetische Kautschuk, seine Homologen und Analogen. Jurjew 1912. (Russisch.)

1066. Richter, V. $v$, Organische Chemie. Bd. II. 11. Auflage. Bonll 1913.

106. Jabresbericht über die Fortschritte der Chemie und verwandter Teile anderer Wissenschaften, begründet von J. Licbig und H. Kopp, herausgegeben von J. Troeger und E. Baur. Für 1909, Heft 13. Braunschweig 1913.

2170. Bujard, A., Die Feuerwerkerei. Berlin und Leipzig 1912.

2171. Múnz, P. und Rosenberg, N., Die richtiggestellten Hauptmethoden der Harnanalyse. Berlin 1913.

2172. Abhandlungen aus dem Institut von E. Beckmann, Laboratorium für angewandte Chemie der Universität Jeipzig, Bd. V, Januar 1910 bis März 1912. Leipzig 1912.

Iu der Sitzung wurden folgende Vorträge gebalten:

1. Berta Heimann und W. Marckwald: Über das Verhältnis ron Uran zu Radium in Uranerzen. - Vorgetragen vou Hru. W. Marckwald.

2. R. Willstätter und M. Heidelberger: Zur Kenntnis des Cyclo-octatetraens. - Vorgetragen von Hrn. R. Willstätter.

3. R. Willstätter: Über die Einführung des Magnesiums in Derivate des Chlorophylls. - Vorgetragen vom Verfasser.

Der Vorsitzende:

W. Will.
Der Schriftführer.

F. Mylius. 